\title{
Two-year comparative study on immediate versus delayed induction in term premature rupture of membranes
}

\author{
John Mary Betty Agnes ${ }^{1}$, Sowmyanarayanan Lavanya ${ }^{2 *}$
}

\begin{abstract}
${ }^{1}$ Department of Obstetrics and Gynecology, Government R. S. R. M Hospital, Chennai, Tamil Nadu, India
${ }^{2}$ Department of Obstetrics and Gynecology, Narayana Medical College, Nellore, Andhra Pradesh, India
\end{abstract}

Received: 25 November 2017

Accepted: 04 December 2017

\section{*Correspondence: \\ Dr. Sowmyanarayanan Lavanya, \\ E-mail: lav_sowgi@yahoo.com}

Copyright: (C) the author(s), publisher and licensee Medip Academy. This is an open-access article distributed under the terms of the Creative Commons Attribution Non-Commercial License, which permits unrestricted non-commercial use, distribution, and reproduction in any medium, provided the original work is properly cited.

\section{ABSTRACT}

Background: Premature rupture of membranes (PROM) has an incidence of about $10 \%$ of all pregnancies and is a significant event as it can cause maternal complications, neonatal morbidity and mortality. Some believe that the expectant management of PROM at term does not increase the perinatal and maternal morbidity, and immediate induction of labour leads to an increased caesarean section rate. There are some authors who report a significant increase in the rates of neonatal, maternal infection and foetal distress if delivery occurs over 24 hours after PROM. Thus, a data is required to manage the cases of PROM to effect safe delivery for both mother and baby. The objective of the study was to compare the neonatal and maternal outcomes between immediate and delayed induction with PG E2 gel in term PROM.

Methods: A hospital based study in women admitted to Obstetrics and Gynaecology Department at R.S.R.M Hospital with a sample of 400 patients in age group between 19- 35 years with gestational age between 37 and 41 weeks were selected for the study. All the 400 cases who presented with term PROM were admitted in labour room and history was elicited regarding age, menstrual and obstetric history with enquiry regarding the time of rupture of membranes, duration and amount of leaking with general, systemic and detailed obstetric examination.

Results: The number of PG E2 gel needed for induction varied between the two groups. Around 45 patients in group 2 needed second dose of gel whereas only 32 patients required the second dose in the late induction group and 72 cases got into active labour. There was no difference in maternal and neonatal infectious morbidity between the two groups. This may be due to the use of prophylactic antibiotics. Neonatal outcome was equally good in both the groups.

Conclusions: Delayed induction of labour in PROM after a waiting period of 12 hours stands as a reasonable option as it reduces the number of operative deliveries without compromising the maternal and neonatal outcome.

Keywords: Amniotic fluid, labour induction, PGE2 gel, Premature rupture of membranes, Operative delivery

\section{INTRODUCTION}

Foetal membrane or the chorioamniotic membrane refers to the chorion and amnion which surround and protect the foetus during pregnancy. Normal progress and outcome of pregnancy depends in part on the normal development and structural integrity of the Foetal membrane. One of its major functions is to maintain the protective intrauterine fluid environment upon which the foetus depends for its survival in utero. In most pregnancies labour begins at term, in the presence of intact foetal membranes. Without interventions the spontaneous rupture usually occurs near the end of the first stage of labour. Premature rupture of membranes (PROM) is defined as the spontaneous rupture of amniotic membrane with a release of amniotic fluid before the onset of 
labour. If the membranes rupture after 37 weeks of gestation it is called term Premature Rupture of Membranes. If the rupture of membranes (ROM) occur before 37 weeks of gestation is termed as the preterm premature rupture of membrane (PPROM). Premature rupture of membrane has an incidence of about $10 \%$ of all pregnancies and is a significant event as it can cause maternal complications, increased operative procedures, neonatal morbidity and mortality. ${ }^{1}$

Some authors like Cammu $\mathrm{H}$ et al believe that the expectant management of premature rupture of membranes at term does not increase perinatal and maternal morbidity, and that an aggressive attitude to premature rupture of membranes with immediate induction of labour leads to an increased caesarean section rate. ${ }^{2}$ There are some authors like Neuhaus W et al who report a significant increase in the rates of neonatal and maternal infection and foetal distress if delivery occurs over 24 hours after premature rupture of membranes. ${ }^{3}$ Immediate induction of labour has shown to reduce the duration of hospitalization and occurrence of neonatal and maternal infection. ${ }^{4}$ Patients with premature rupture of membranes who were induced within 6 hours of rupture of membranes, $90 \%$ delivered within 24 hours of rupture of membrane as compared to group managed expectantly in which $60 \%$ delivered within 24 hours. $^{5}$ Also, with increasing time since the rupture of membranes to delivery, a higher incidence of histological chorioamnionitis was observed in some studies. ${ }^{6}$ The causes of PROM are bacterial vaginosis, genitourinary infections, polyhydramnios, multiple pregnancies, malpresentations, CPD. ${ }^{7}$ The risk factors are black race, low socio economic status, history of second trimester termination previously, smoking and previous PROM. Pathogenesis behind PROM is choriodecidual infection and inflammation leading to a cascade of leucocyte activation and cytokine release resulting in premature cervical ripening and possible membrane rupture. ${ }^{8}$ The longer the time between membrane rupture and delivery, the greater the risk of infection, especially if vaginal examinations are performed frequently. ${ }^{9}$

Complications of PROM are chorioamnionitis, cord prolapse, abruptio placenta, oligohdramnios, neonatal sepsis, birth asphyxia, deformities and distress. Steroids are given in PPROM to effect foetal lung maturity. Tocolysis is offered only for the purpose of steroids to cover up for lung maturity and to prolong the time to refer them to a tertiary care hospital with neonatal intensive care facilities. The management of PROM is either expectant or immediate intervention. Immediate intervention means effecting induction of labour with $\mathrm{PGE}_{2}$ gel to make the Bishop's score favourable and effect delivery. Expectant management is to wait for spontaneous onset of labour and deliver under cover of antibiotics and monitoring. The immediate induction may require more than a single dose of PGE2 gel and increase the operative delivery. On the other hand, the infection rate increases if the delivery occurs after 24 hours after rupture of membranes. At term, nearly $80 \%$ of women go into labour within the first 24 hours after rupture of membranes. ${ }^{10}$ Evidence indicates that pre-term membranes are stronger than term membranes and indeed PROM occurs in $10 \%$ of pregnancies at term while in only $0.7-2 \%$ of pregnancies before 37 completed weeks. The relative rarity of pre-term PROM has prompted investigators to examine the physical properties of the Foetal membranes in order to determine whether rupture is caused by an inherent weakness of the membrane material or by local defects in the membrane structure.

The aim and purpose of this study was to compare the maternal and neonatal outcomes between immediate and delayed induction (after 12 hours) with PGE2 gel in women with term premature rupture of membranes (PROM). It was conducted in women admitted in the labour room at Govt RSRM hospital.

\section{METHODS}

A hospital based comparative study involving women admitted in Department of Obstetrics and Gynaecology at Government R.S.R.M Hospital for a period of two years between June 2015 and May 2017. A total of 400 cases of age group between 19 and 35 years with rupture of membranes prior to onset of labour with the gestational age between 37 and 41 weeks were selected for the study.

\section{Inclusion criteria}

- Term premature rupture of membranes $<12$ hours duration at the time of admission

- No evidence of foetal distress

- No evidence of sepsis (maternal tachycardia, pyrexia, uterine tenderness)

- No other risk factors in pregnancy e.g. medical complications, malpresentation, abnormal lie, multiple pregnancy and previous caesarean section

- Modified Bishops score <6

- All neonates born to women included in the study.

\section{Exclusion criteria}

- Premature rupture of membranes for $>12$ hours at the time of admission

- Gestational age <37 weeks, >41 weeks

- Evidence of foetal distress/sepsis.

- Medical complications, malpresentation, abnormal lie, multiple pregnancy and previous caesarean section

- $\quad$ Suspected CPD

- Women in active labour

- Patients who are HIV positive or immune compromised.

All the 400 cases who presented with term PROM were admitted in labour room and a detailed history was elicited regarding age, menstrual and obstetric history 
with detailed enquiry regarding the exact time of rupture of membranes, duration and amount of leaking. It was followed by general, systemic and detailed obstetric examination. A sterile speculum examination was done, and PROM confirmed by visualisation of amniotic fluid from cervical os or posterior fornix. A high vaginal swab was taken for culture. Pelvic examination was done to assess the modified Bishop's score. Cases satisfying the inclusion criteria were classified into two groups:

- Early induction group (PROM - admission interval $<6$ hours) and,

- Delayed induction group (PROM - admission interval 6-12 hours).

Early induction group was induced immediately after admission with $\mathrm{PGE}_{2}$ gel and delayed induction group was induced after 12 hours after PROM. All women were monitored with prophylactic antibiotics, NST and temperature chart. If Bishop's score was unfavourable second dose of gel was kept. If score was favourable augmentation of labour was done with oxytocin.

The neonates born to the women in the study were examined by the paediatrician immediately after birth and then once daily. Symptoms and signs of neonatal sepsis were looked for. A sepsis screen was performed (TC, platelet count, CRP).

All the neonates who were screen positive (any one test) were subjected to blood culture and sensitivity and were given antibiotics (Inj. Ciprofloxacin, Inj. Amikacin) for 5 days. If culture positive, sensitive antibiotics were given for 15 days. Early onset neonatal sepsis (i.e. sepsis within 72 hours of birth) is attributed to exposure to bacteria in the antepartum and peripartum period. Late onset neonatal sepsis (more than 72 hours after birth) is usually nosocomial and hence not related to PROM.

The following outcomes were compared between the two groups.

- PROM - delivery interval

- No. of PGE2 doses

- Mode of delivery

- Newborn depression (1 - min and 5 - min APGAR scores)

- Neonatal sepsis

- Maternal morbidity

- Duration of hospital stay

\section{RESULTS}

Of the total number of deliveries in 2015 to 2017, there were 1123 cases of term PROM (incidence: 5.2\%).

After applying the inclusion and exclusion criteria, there were 400 cases eligible for the study. 200 were analysed in group A and 200 in group B.

\section{Analysis of obstetric score (parity)}

For comparability, 140 primigravida and 60 multigravidas were included in each group. G2A1 were included in the primigravida.

\section{Analysis of gestational age}

The patients with gestational ages ranging from 37 to 41 weeks were included in the study. The patients in both groups were comparable with regard to gestational age (P: 0.562). The mean GA in both groups was 38 weeks.

\section{Analysis of number of $P G E_{2}$ doses}

72 cases in the delayed induction group entered active labour during the waiting period. They did not require induction. Significantly higher doses of $\mathrm{PGE}_{2}$ ( $\mathrm{p}$ value 0.00 ) were required in the immediate induction group as compared to the delayed induction group (155 versus 96). 45 patients in the immediate induction group needed 2 doses of PGE2 while only 32 in the delayed induction group needed 2 doses (Table 1).

Table 1: Analysis of number of $\mathrm{PGE}_{2}$ doses.

\begin{tabular}{|lll|}
\hline No. of PGE 2 doses & $\begin{array}{c}\text { Group A } \\
(\mathbf{n}=200)\end{array}$ & $\begin{array}{l}\text { Group B } \\
(\mathbf{n}=200)\end{array}$ \\
\hline 0 & 0 & 72 \\
\hline 1 & 155 & 96 \\
\hline 2 & 45 & 32 \\
\hline
\end{tabular}

Pearson Chi-Square P-value $=0.000$ (significant)

\section{Prom-delivery interval}

Most of the patients (48\%) delivered within 14 to 20 hours of PROM. The earliest PROM-delivery interval was 8 hours (one patient in early induction group). One of the patients in the delayed induction group had the longest PROM-delivery interval of 30 hours. More number of patients $(78 \%)$ in the early induction group delivered within 14 hours of PROM as compared to the delayed induction group. The PROM-delivery interval was significantly more in the delayed induction group as compared to the early induction group (statistically significant: Pearson chi P value: 0.00) (Table 2).

Table 2: PROM delivery interval.

\begin{tabular}{|lll|}
\hline $\begin{array}{l}\text { PROM-delivery } \\
\text { interval }\end{array}$ & $\begin{array}{l}\text { Group A } \\
(200)\end{array}$ & $\begin{array}{l}\text { Group B } \\
(200)\end{array}$ \\
\hline 8-14 hours & 98 & 38 \\
\hline 14-20 hours & 92 & 98 \\
\hline$>20$ hours & 10 & 64 \\
\hline
\end{tabular}

\section{Mean prom-delivery interval}

Mean PROM delivery interval in group A was 14.58 hours whereas in group B 18.79 hours showing early delivery in early induction group. 


\section{Mode of delivery}

There were more number of caesarean sections in the early induction group when compared to the delayed induction group which was statistically significant $(\mathrm{P}$ value: 0.049 ). The percentage of operative vaginal deliveries were almost the same in both groups (Table 3 ).

Table 3: Mode of delivery.

\begin{tabular}{|llll|} 
& $\begin{array}{l}\text { Group A } \\
(200)\end{array}$ & $\begin{array}{l}\text { Group B } \\
(200)\end{array}$ & P value \\
\hline NVD & 106 & 130 & \\
\hline Forceps & 20 & 18 & \\
\hline Ventouse & 12 & 10 & \\
\hline LSCS & 62 & 42 & 0.049 \\
\hline
\end{tabular}

\section{Indications for LSCS}

$71 \%$ of LSCS done in group B were for foetal distress as compared to $45 \%$ in group A. There were significantly more failed inductions and labour abnormalities in group A when compared to group B (Table 4).

Table 4: Indications for $\operatorname{LSCS}(n=104)$.

\begin{tabular}{|lll|}
\hline Foetal distress & Group A (62) & Group B (42) \\
\hline $\begin{array}{l}\text { Failed induction } \\
\begin{array}{l}\text { Non-progression } \\
\text { of labour }\end{array}\end{array}$ & $18(31 \%)$ & $30(71.4 \%)$ \\
\hline
\end{tabular}

\section{Maternal morbidity}

Infective morbidity was similar between the two groups ( $\mathrm{P}$ values $>0.05$ not significant $)$.

\section{APGAR score less than 7}

16 neonates in group A and 20 in group B had 1-minute APGAR of less than 7. One neonate in group A and 3 in group B had 5-minute APGAR of less than 7. There is no statistically significant difference in both.

\section{DISCUSSION}

$\mathrm{P}$ Immediate induction was compared with that of delayed induction after 12 hours of PROM in term PROM cases. Both study groups were comparable with regard to age, parity, booking status and gestational age.

During the waiting period of 12 hours $38 \%$ of cases entered active labour in the delayed induction group. So significantly lesser number of patients in the delayed induction group required induction compared to early induction group. The results were similar to that of Krupa et al which showed that significantly higher doses of $\mathrm{PGE}_{2}$ were required in immediate induction group. ${ }^{11}$ This is comparable to the following studies: Dare et al: $50 \%$ (in 12 hours), Krupa et al: $80 \%$ (in 24 hours) and Poornima et al: $60 \%$ (in 12 hours). ${ }^{12,13}$

Significantly higher doses of PGE2 were required in the early induction group.

The PROM delivery interval was significantly shorter in the early induction group. Bangal et al and Alcalay et al also inferred that mean period from rupture of membrane to delivery was shorter significantly in the induction group compared to the expectant group. ${ }^{14}$

LSCS and operative vaginal deliveries were more in the early induction group. In this study, the caesarean section rate was significantly higher in the early induction group compared to the delayed induction group (31\% versus $21 \%, \mathrm{P}$ value $=0.049$, significant). In this aspect, our inference was different from that of Krupa et al and Alcalay et al who showed similar rates of normal and caesarean deliveries between the two groups. The results of Poornima et al were comparable to that of the present study. The difference in LSCS rate was largely due to the increased incidence of labour abnormalities and failed induction in the immediate induction group. The number of operative vaginal deliveries was also higher in the early induction group. This is also the inference of Alcalay et al. ${ }^{15}$

Failed induction and labour abnormalities were more in the early induction group (statistically significant), while foetal distress was slightly higher in the delayed induction group. Either non-reassuring CTG or meconium stained liquor were considered as foetal distress.

There was no significant difference in chorioamnionitis in both the groups. Leukocytosis was more specific marker compared to fever and maternal tachycardia. Several studies show that chorioamnionitis is reduced with the use of prophylactic antibiotics at term Fever appeared to be a nonspecific marker, while leucocytosis was more specific. No case had foul smelling vaginal discharge or uterine tenderness.

There was no difference in maternal and neonatal infectious morbidity between the two groups. This may be due to the use of prophylactic antibiotics. Maternal morbidity was analysed between the two groups by taking into consideration the number of patients who had urinary tract infection, LSCS site wound infection and positive high vaginal swab culture.

Neonatal outcome was equally good in both the groups. All neonates were screened for sepsis using total count, platelet count and C-reactive protein. All neonates were discharged in healthy condition, but the culture positive babies were given IV antibiotics for 15 days. Klebsiella, E. coli and Staphylococci were the isolated pathogens. Though the mean hospital stay was not different, more 
number of patients in group A had a stay of $>5$ days due to increased number of LSCS.

\section{CONCLUSION}

To conclude delayed induction after a waiting period of 12 hours stands as a reasonable option in term PROM and it decreases the number of operative deliveries without compromising the maternal and neonatal outcome.

\section{Funding: No funding sources}

Conflict of interest: None declared

Ethical approval: The study was approved by the Institutional Ethics Committee

\section{REFERENCES}

1. Berkowitz GS, Papiernik E. Epidemiology of preterm birth. Epidemiol Rev. 1993;15:41-8

2. Cammu H, Verlaenen H, Derde MP. Premature rupture of membranes at term in nulliparous women a hazard Obstet Gynaecol. 1990;76:671-4.

3. Neuhaus W, Eibach HW, Ahr A, Bolte. PROM problems and obstetric management. Obstet Gynecol. 1993;53:843-8.

4. Duff P, Huff P, Gibbs RS. Management of PROM and unfavourable cervix in term pregnancy. J Obstet Gynaecol. 1984;63:697-702.

5. Russel KP, Anderson GVT. The aggressive management of ruptured membranes. Am J Obs Gynaecol. 2000;83:930.

6. Lee T, Carpenter M, Heber WW, Silver HM. Preterm premature rupture of membranes: risks of recurrent complications in the next pregnancy among a population-based sample of gravid women. Am J Obstet Gynecol. 2003;188:209-13.

7. Harger JH, Hsing AW, Tuomala RE. Risk factors for preterm premature rupture of fetal membranes: a multicenter case-control study. Am J Obstet Gynecol. 1990;163:130-3

8. Lockwood CJ, Wein R, Chien D. Fetal membrane rupture is associated with the presence of insulin-like growth factor-binding protein-1 in vaginal secretions. Am J Obstet Gynecol. 1994;171:146-50.

9. ACOG Committee on Practice Bulletins-Obstetrics, authors. Clinical management guidelines for obstetrician-gynecologists. (ACOG Practice Bulletin No. 80: premature rupture of membranes). Obstet Gynecol. 2007;109:1007-19.

10. Hannah ME, Ohlsson A, Farine D. Term PROM study group, authors. Induction of labour compared with expectant management for prelabor rupture of the membranes at term. $\mathrm{N}$ Engl $\mathrm{J}$ Med. 1996;334:1010.

11. Shah K, Joshi H. Premature rupture of membrane at term: early induction versus expectant management. J Obstet Gynaecol India. 2012;62(2):172-5.

12. Dare MR, Middleton P, Crowther CA. Planned early birth versus expectant management (waiting) for prelabour rupture of membranes at term (37 weeks or more). Cochrane Database Syst Rev. 2006; 1:CD005302.

13. Poornima B, Reddy DDB. Premature rupture of membranes at term: immediate induction with pge2 gel compared with delayed induction. J Obstet Gynecol India. 2011;61(5):516-8.

14. Bangal VB, Gulati P, Shinde KK, Borawake SK. Induction of labour versus expectant management for premature rupture of membranes at term. Int $\mathbf{J}$ Biomed Res. 2012;3:164-70.

15. Alcalay M, Hourvitz A, Reichman B, Luski A, Quint J, Barkai G, et al. Prelabour rupture of membranes at term: early induction of labour versus expectant management. Eu J Obstet Gynecol Reprod Biol. 1996;70(2):129-33.

Cite this article as: Agnes JMB, Lavanya S. Twoyear comparative study on immediate versus delayed induction in term premature rupture of membranes. Int J Reprod Contracept Obstet Gynecol 2018;7:94-8. 